\title{
UNA NOTA MANUSCRITA DE FRANCISCO DE TOLEDO, S.I., SOBRE LA CONSTRUCCIÓN DE LA IGLESIA DE IL GESÙ
}

\author{
POR \\ MACARENA MORALEJO ORTEGA \\ Escuela Española de Historia y Arqueología de Roma
}

El descubrimiento de un documento inédito acerca de la construcción de la iglesia de $I l$ Gesù proporciona nuevos datos acerca de los cambios en el diseño planimétrico y urbanístico de la zona. El manuscrito presumiblemente fue redactado por el Cardenal Francisco de Toledo, S.I., en el año 1576.

The discovery of an unpublished document dealing with the construction of the church of Il Gesú provides new information concerning the changes in the planimetric and urbanistic design of the area. The manuscript presumedly was written by Cardinal Francisco de Toledo S.I. in 1576.

El hallazgo de un documento inédito, fechado en 1576, sobre la construcción de la primera iglesia de la Compañía de Jesús en el mundo ofrece novedosos datos sobre el proceso de edificación de $I l$ Gesù y el explícito respaldo de las altas jerarquías eclesiásticas a la incipiente Orden fundada por San Ignacio de Loyola.

El material de archivo publicado por el profesor Pio Pecchiai ${ }^{1}$, S.I., en su monografía sobre Il Gesù confirma precisamente la ausencia de documentación sobre su construcción entre 1577 y $1581^{2}$, de ahí la importancia de esta nota manuscrita sobre la construcción del templo en noviembre de1576.

Este desconocido documento se encuentra en el reverso de la última página del sermón ${ }^{3}$ predicado por el jesuita Francisco de Toledo con este encabezamiento: Predica del reverendo

\footnotetext{
* Mi agradecimiento al R.P. Heinrich W. Pfeiffer, director del seminario La Chiesa del Gesù en la Pontificia Università Gregoriana de Roma por sus orientaciones bibliográficas en la redacción de este artículo. Agradezco también al R.P. Silvano Giordano O.C.D., docente en el mismo centro universitario, sus consejos en el tratamiento de textos.

${ }^{\prime}$ Cfr. Pecchiai P. S.I. (con prefazione del P. Pietro Tacchi Venturi S.I.) Il Gesù di Roma, ed. Società Grafica Romana, Roma, 1952. Esta monografía es la única publicación que se ha editado con material de archivo.Véase también De Buck V., Le Gesù de Rome. Notice descriptive et historique, Vandereydt, 1871.

${ }^{2}$ Cfr. Pecchiai P., op.cit., pp.74: Il registro della fabbrica sul quale abbiamo fondato questa cronistoria giunge fino al 1581, ma le notizie sul progredire dell'edificio si arrestano al 1577.

${ }^{3}$ Cfr. BAV, Barb. Lat. 5377, ff 17-20. El tema del sermón aparece enunciado por el predicador Francisco de Toledo S.I. en el encabezamiento: Beati estis cum male dixerint vobis (homines) et persecuti vos fuerint et dixerint omne malum adversum vos, mentientes, propter me; gaudete et esultate, quoniam merces vestra copiosa est in [sic] (Mt, 5, 11-12).
} 
Dottore Francisco Toledo discepolo del Gesù fatta nella sala di Costantino al Palazzo Vaticano innanti Sua Santità la Domenica fra l'ottava di tutti i santi I'anno 1574.

La prédica, redactada de puño por el Padre Toledo, no es la única conservada en el volúmen utilizado que reúne otros sermones del mismo jesuita fechados entre 1572 y 1576 . En el caso que nos ocupa, la homilía comenta el capítulo 5, versículo 11 del Evangelio de San Mateo: «Dichosos seréis cuando os injurien, os persigan y digan contra vosotros toda suerte de calumnias por culpa mia» ${ }^{4}$.

La semejanza entre la grafía de sus sermones y la extensa nota de carácter más privado sobre la construcción de Il Gesù, asi como la proximidad cronológica entre la documentación conservada en el mismo volumen, hacen suponer como autor de ambos textos al Padre Toledo.

La ausencia de estudios críticos sobre la producción teológica de Francisco de Toledo ${ }^{5}$, ilustre predicador en la corte pontificia de la segunda mitad del Xvi y primer Cardenal de la Compañía de Jesús, ha infravalorado su extraordinaria contribución a la Historia de la Iglesia. La inexistencia de una publicación de carácter monográfico que analice su prestigio como teólogo, diplomático y orador dificulta el análisis de conjunto de su producción teórica ${ }^{6}$.

Su nacimiento en Córdoba hacía 1530 en el seno de una familia de origen hebreo, no fue, sin embargo, un obstáculo para madurar su vocación sacerdotal e ingresar en la Compañía hacia 1550. Cursó estudios en artes en la Universidad de Zaragoza, para trasladarse posteriormente a Salamanca, donde completó su formación junto al eminente teólogo Domingo de Soto O.P. llegando incluso a enseñar en el curso académico 1557-1558 en la citada ciudad castellana.

En la primavera de 1559 fue enviado por la Compañía de Jesús a Roma para formar parte del prestigioso claustro de catedráticos del Collegio Romano ${ }^{7}$ como maestro de filosofía y teología.

El nombramiento del Padre Toledo como Teólogo de la Sagrada Penitenciaria y predicador del Papa y del Sacro Colegio Cardenalício en 1569 permitió al jesuita ejercer su cargo durante mas de veinte años.

Sus sermones, en su mayoría inéditos, expresan perfectamente el espíritu crítico presente en las directrices del Concilio de Trento junto a la voluntad explícita de convencer predicando, como elocuentemente había sugerido la literatura teológica de la Contrarreforma.

Este acercamiento a la corte papal, durante el pontificado de Gregorio XIII, Sixto V y en especial bajo Clemente VIII ${ }^{8}$, agravó la conflictiva relación del Padre Toledo con la Compañía de Jesús. La enemistad tuvo su punto culminante con la elección, por parte del cordobés,

\footnotetext{
${ }^{4}$ Los sermones fueron redactados en lengua toscana, idioma utilizado en la predicación pública. El contenido de la plática se reseñaba en latín mediante la selección a priori de un versículo bíblico.Véase nota 3 para el caso que nos ocupa.

${ }^{5}$ Cfr. Sommervogel C., Bibliotheque d'écrivains de la Compagnie de Jésus, vol. VIII, pp. 64-82, Paris, 1893 y O’Neill C.E. S.I.-Domínguez J.M ${ }^{a}$, Diccionario Histórico de la Compañía de Jesús, vol. IV, pp.3807-3808, Roma-Madrid, 2001.

${ }^{6}$ Las maquiavélicas actuaciones políticas de la alta jerarquía eclesiástica que rodeaba a Francisco de Toledo han sido descritas en la publicacion de Klaus Jaitner, Die Hauptinstruktionen Clemens' VIII. für die Nuntien und Legaten an den europäischen Fürstenhöfen 1592-1605 (Instructiones Pontificum Romanorum), Tübingen, 1984, pp. LXXXVIII.

7 El incremento del alumnado en el Collegio Romano en el último cuarto del siglo XVI como escribió Roberto Bellarmino S.I. en 1593, con la presencia de unos 2000 discípulos y 200 jesuitas, produjo un ascenso en el número de profesorado, mayoritariamente de origen español. Jesuitas como Miguel Vázquez de Padilla, Alfonso de Salmerón o Bartolomé del Alcázar continúan la trayectoria profesional del Padre Toledo. Véase Pérez Goyena A., Catedráticos de teología españoles en Roma, en Estudios Eclesiásticos, 1926, pp.26-43 y Gómez Hellin L. Toledo, lector de filosofía y teología en el Colegio Romano, Archivo Teológico Granadino, 3, 1940, pp.1-18.

${ }^{8}$ El epitafio del Cardenal Toledo en la Basílica romana de Santa María La Mayor, alude precisamente a su prestigio como predicador. El deficiente estado de conservación de la sepultura no permite leer integramente la inscripción: DOM / Franciscus Toletus Cordubensis Presb. Card. Summus Theologus Verbi Dei Praedicator eximius in rebus magnis agendis consilio et prudentia singulari qui ob excellentem virtutem et merita praeclara Clementis VIII Pont. Max. IV Diei [?] Primus in Societatem Iesu amplissimam dignitatem induit. Vixit annos LXIII Menses XI Dies X. Obiit anno MDXCVI die XIV Septembris. S. Dei genitrice [?] aere de instituta presbyteris qui ad eius altare missas celebrarent censum perpetuo attribui iussit. Benedictus Iustinianus et Petrus Aldobrandinus cardd. executores testamentarii collegae optimo et capitulum et canonici huius basilicae viro amplissimo et optime de se merito posuerunt.
}

AEA, LXXVI, 2003, 302, pp. 169 a 176 
de su sepultura en la basílica de Santa Maria la Mayor, decisión que no fue secundada por otros jesuitas de su generación y rango que optaron por enterrarse en Il Gesù.

La confianza depositada por el papado en el cardenal cordobés, le permitió jugar una baza importante en la política internacional ${ }^{9}$, siendo enviado por los pontífices a misiones especialmente complejas ante el rey Segismundo II de Polonia, en la corte de Maximiliano II en Viena y apoyando la conversión al catolicismo de Enrique IV de Navarra y Francia.

Su lucha contra la herejía, como testimonian sus denodados esfuerzos para forzar la sumisión de Michel de Bay (Baius) ${ }^{10}$, se puso de manifiesto, asimismo, en sus sermones en los que lanzaba duros ataques contra los católicos disidentes.

Este criterio expositivo y crítico, presente también en sus exégesis bíblicas y comentarios sobre Aristóteles ${ }^{11}$, se repite en la redacción de sus prédicas, como así lo atestigua el documento encontrado.

En el manuscrito, presumiblemente autógrafo del jesuita, se describe el aparato litúrgico de tres celebraciones eucarísticas en noviembre de 1576 en el interior de la iglesia de Il Gesù, en aquel momento, aún en obras.

En este sentido, al interés de los actos religiosos, el contenido de los sermones y la asistencia de importantes miembros del estamento eclesiástico, habría que añadir las importantes noticias que el manuscrito proporciona para la historia del arte.

Junto a la descripción de los feligreses reunidos en la renovada Piazza degli Altieri se revelan las modificaciones del trazado urbanístico ${ }^{12}$ con la construcción ex novo de la iglesia de Il Gesù.

La delineación en el documento de la Piazza degli Altieri como un espacio amplio ocupado en toda su extensión por los denominados en el escrito como «cocchi», es decir, carrozas, alude a la paulatina modificación del espacio urbano, en aquel momento parcialmente reformado, con la destrucción de los palacios de la familia Altieri y de la parroquia del Rione della Pigna que ocupaba el sector más central de la capital.

En ese sentido el uso de la parroquia del citado Rione por parte de la Compañía, resultó proverbial para el impulso de la Orden en el ámbito romano. La iglesia, conocida como Santa Maria della Strada y centro de peregrinación en Roma, fue cedida por el papa Pablo III en 1541 a los jesuitas ${ }^{13}$, aunque dada la limitada capacidad del templo, el fundador de la Orden decidió promover la construcción de la citada iglesia de Il Gesù.

La Compañía de Jesús modificó espacialmente la parroquia con la creación de dos nuevas naves, aprovechando el reducido espacio no construido en torno a la iglesia. El tamaño del templo se vió ampliado, aunque no bastaba para albergar a los fieles en los días festivos.

Además, la iglesia había adquirido un significado muy especial para la Compañía, ya que

\footnotetext{
${ }^{9}$ El papel jugado por el P.Toledo en la política internacional se pone de manifiesto en Roca M., Documentos inéditos en torno a Miguel Bayo, Anthologica Annua I, 1953, pp. 303-476; Cozzi G., Un documento sulla crisi della «sacra lega»: Le confidenze del Padre Francisco de Toledo all'Avogadore di Comun Nicolò Barbarigo, Archivo Veneto 67 (1960) pp. 76-96.

${ }_{10}$ Roca M., op.cit.

"Su obra teológica más importante se publicó con el título: Summa de instructione sacerdotum en 1599, superándose las setenta ediciones en toda Europa. En cuanto a sus escritos de historia de la filosofía destacan las obras: Introductio in Dialecticam Aristotelis (Roma, 1561) y Comentaria in universam logicam (Roma, 1572). La contribución de Toledo al renacimiento del pensamiento aristotélico se expone en Lohr H., Latin Aristotle Commentaries II: Renaissance authors, Firenze, 1988, pp. 458-464.

Un elenco detallado de las publicaciones de Toledo y sus manuscritos inéditos en Sommervogel, C., op.cit., pp. 64-82.

12 Véase el artículo publicado por Pietro Pirri, S.I., La topografía del Gesù di Roma e le vertenze tra Muzio Muti e S. Ignazio secondo nuovi documenti, en Archivium Historicum Societatis Iesu, vol. X, 1941, pp. 177-217.

${ }^{13}$ Las ediciones posteriores a 1541 de la guia Mirabilia Urbis Romae con el anexo Antichità di Roma de Andrea Palladio confirman ya el traspaso de dirección del templo a la Compañía de Jesús. Para la literatura topográfica de este periódo véase Schlosser von J., La literatura artística, Ed. Cátedra, Madrid, 1976, pp. 200 y ss. (presentación y adicciones del prof.Antonio Bonet Correa).
} 
se había enterrado a San Ignacio de Loyola ${ }^{14}$, San Francisco de Borja, Diego Laínez y a otros jesuitas de la primera generación con la esperanza de trasladar sus restos a la anhelada iglesia de Il Gesù.

Estas transformaciones obligaron, ya en 1568, a permitir la ocupación de la calle que pasaba delante de esta iglesia, distorsionando el entramado viario, para posteriormente destruirla conservando únicamente la imagen de la Virgen en el nuevo templo.

La iglesia, conocida popularmente como la Madonna della Strada, aludiendo a la presencia de una imagen bizantina en su altar, se denominó como iglesia de Santa Maria degli Altie$r i$ ya que se encontraba haciendo esquina con el palacio de la familia.

La alteración fundamental del espacio urbano en esta manzana se produjo con la definitiva tercera fundación ${ }^{15}$ de la iglesia de $I l$ Gesù en 1568 con la participación de los arquitectos Giovanni Tristano y Giacomo Barozzi (Vignola). En el mismo año, las conversaciones entre el Papado, la Compañía y los denominados Maestri delle Strade ${ }^{16}$ dispusieron la adquisición del ruinoso palacio de Girolamo Altieri, cuya compra permitiría a la Compañía la construcción de dos tercios de su nueva iglesia. El complejo intricado de calles, huertos, graneros, callejones, y palacios alteraba la disposición de la hipotética iglesia, que, de cualquier manera, debía tener, al menos, una orientación frontera con dirección hacia el Palacio de San Marco.

Se solicitaba, asimismo, el cierre de uno de los callejones que discurría entre la casa Picensanti y el palacio Altieri, para poder comenzar las obras, que debían alcanzar el palacete de la familia Astalli ${ }^{17}$, todavía reacia a la venta.

Las casas, adquiridas a las familias que habían vivido en la zona, fueron demolidas atendiendo a las necesidades constructivas de la iglesia aprovechando incluso los materiales de desecho tras el derrumbamiento. La destrucción de los inmuebles se realizó según iba avanzando la construcción, combinando las labores de desescombro y alzamiento.

El documento encontrado refleja el avanzado estado de las obras, aunque, seguramente, sufrieron un receso hasta $1581^{18}$, ya que los obreros debían estar ocupados con las labores de limpieza y allanamiento de toda el area referida. El manuscrito refiere claramente el cierre de la antigua parroquia, que todavía estaba en pie: «[‥] Da qui innanzi dunque seguitarono quei

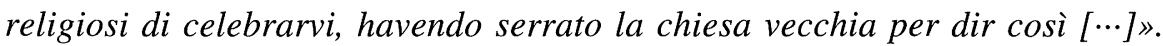

Estas transformaciones, no habrían tenido sentido si la Compañía hubiese elegido construir su iglesia junto a la Casa Profesa, en la denominada Via Capitolina (hoy Aracoeli) otorgando a su nuevo templo un enclave quizás de segundo orden respecto al que finalmente se eligió. El Cardenal Farnese, mecenas de la obra, optó por una ubicación en la que la fachada de la iglesia jugaba con la perspectiva de la calle Cesarini, la más importante, que desembocaba en la Piazza degli Altieri, y conducía, en el lado septentrional al Palacio e Iglesia de San Marcos, residencia temporal del pontífice.

\footnotetext{
${ }^{14}$ Tras el fallecimiento de San Ignacio de Loyola el 31 de julio de 1556, su cuerpo fue depositado en un sencillo sepulcro en la parroquia de Santa Maria della Strada. En 1587 los restos del vizcaino fueron trasladados a la iglesia de Il Gesù habiéndose demolido la antigua iglesia por las obras.

${ }^{15}$ Con anterioridad al óbito del fundador de la Compañía en 1556 se había intentando levantar una iglesia en los aledaños de Piazza San Marco. La primera fundación frustada se produjo en 1550 con la promoción de Alfonso de Villalobos y planta diseñada por el arquitecto Nanni di Baccio; el segundo patronato fue una iniciativa del cardenal Bartolomé de la Cueva con diseño planimétrico de Miguel Angel Buonarroti.

${ }^{16}$ Se trataba de cargos públicos que estudiaban las modificaciones del trazado urbano emitiendo un parecer de consenso que debía ser respetado.

${ }^{17}$ La reticencia de la familia Astalli a vender su palacio para trasladarse a otra residencia se plasma perfectamente en esta ingeniosa epístola del patriarca dirigida a los jesuitas: " [ ‥] et fate che in Roma non s'exclami di voi come fece Ennio( $i$ ) poeta in tempo di Nerone che disse: O Romani, andate ad abitare alli Vienti (Veienti) che in Roma se ne fa una casa; et di voi non si dica: O Romani, andate ad habitare ad Frascati che la Compagnia di Jesu di Roma ne fa una casa». Cfr. Pecchiai, P., op.cit., p. 21.

${ }^{18} \mathrm{Se}$ ha mencionado el retroceso en el proceso de construcción entre 1577 y 1581 . Véase nota 2.
}

$A E A$, LXXVI, 2003, 302, pp. 169 a 176 
En el manuscrito aparece ya la necesidad de adecuarse a un proyecto cerrado, ya que se describe la iglesia con un alzado todavía a la mitad de las obras con una construcción desde la fachada al ábside, en la que únicamente se había llegado al tramo del transepto. Ya se había proyectado en planta la comunicación con las estancias de los religiosos mediante la instalación de una puerta de ingreso pequeña desde el exterior y un acceso de reducidas dimensiones desde el interior a las habitaciones de los residentes.

Esta detallada descripción en el manuscrito muestra la ausencia de cambios efectivos en la ejecución de la planta programada, señalando el ingreso en una hipotética cuarta capilla de la banda derecha que aún no existía por la ausencia de cierre en el crucero.

Junto a esta detallada exposición del ambicioso proyecto arquitectónico de la Compañía de Jesús, el inédito documento aporta datos sobre el interés que despertaba la construcción entre las altas jerarquías eclesiásticas.

La anotación manuscrita en el reverso del sermón permite cotejarlo con el texto de la consagración oficial e informarnos de la verosímil asistencia de Francisco de Toledo a ambos actos, la inauguración del templo en 1584, y a las ceremonias descritas, probablemente de su mano, en noviembre de 1576. El único personaje que con certeza asistió a ambas funciones litúrgicas fue Monseñor Paolo Odescalchi ${ }^{19}$, miembro de la distinguida familia romana y obispo de las ciudades de Penne y Adria. La intervención de este prelado en ambos actos confirma el prestigio otorgado a la Compañía con la protección de la nobleza e incluso la intervención en las ceremonias religiosas del propio Gregorio XIII ${ }^{20}$, quien jugó un papel determinante en la expansión de la Compañía de Jesús.

Junto al pontífice aparecen citados destacados nombres de la curia papal como el mecenas y fundador de la iglesia de Il Gesù, el Cardenal Alessandro Farnese, al que se alude como patrono del templo con la indicación de una inscripción ${ }^{21}$, todavía hoy presente, señalando su fundación. Se menciona igualmente su papel como impulsor de las obras en la última linea del documento señalando que en la fachada de la iglesia se citaba de forma explícita su mecenazgo con la colocación de su nombre: Ascanius Farnesius [sic] ${ }^{22}$.

La precedencia en la entrada del templo, en términos protocolarios ${ }^{23}$, se la concede el Cardenal Alessandro Farnese al cardenal Giovanni Morone ${ }^{24}$, nombrado con esta distinción eclesiástica en 1542 por Pablo III.

\footnotetext{
${ }^{19}$ En el documento original que informa sobre la consagración oficial de la iglesia del Santísimo Nombre de Jesús aparece manuscrito:

[‥ ] assistentibus mihi in ipsius Ecclesiae consecratione Reverendis in Christo fratibus, Dominis Thoma Golduello Asaphensi [ $\cdots]$ Paulo Odescalco Pennensi et Adriensi[ $\cdots]$ quorum singuli etiam altaria consecrarunt [ $\cdots]$.

${ }^{20}$ Gregorio XIII ocupó el solio pontificio entre 1572 y 1585 . Procedía de la familia de los Boncompagni y durante su papado realizó innumerables reformas en el campo de la diplomacia, estableciendo las nunciaturas permanentes y fundando el Colegio Romano de la Compañía de Jesús, de cuya dirección se ocupaba la misma Orden.

${ }^{21}$ Cfr. Pecchiai P., op.cit. pp.242: La lapide sulla porta maggiore è un doveroso ricordo del fondatore della chiesa, del quale così parla la epigrafe che vi è scolpita: ALEXANDER FARNESIUS / CARD. S.R.E. VICECANCELL./ PAULI III.PONT.MAX NEPOS/ CUIS AUCTORITATE SOCIETAS/ IESU RECEPTA PRIMUM FUIT / ET DECRETIS AMPLISS. ORNATA / TEMPLUM HOC SUAE MONUMENTUM / RELIGIONIS ET PERPETUAE / IN EUM ORDINEM VOLUNTATIS / A FUNDAMENTIS EXTRUXIT/ ANNO IUBILAEI M. D. LXXV.

${ }^{22}$ Se observa un error en la ortografía del documento: el cardenal no es Ascanio Farnese, como aparece en el texto, sino Alessandro Farnese, como se indica claramente en la inscripción de la fachada de $I l$ Gesù.

${ }^{23}$ Giovanni Morone precede en la entrada al templo al Cardenal Farnese por su condición de obispo de Ostia y por tanto jefe del Colegio Cardenalicio.

${ }^{24}$ El cardenal Morone nace en Milán en 1509 y fallece en Roma en 1580. Era hijo de Gerolamo Morone, futuro gran canciller del ducado de Milán y de Amabilia Fisigara, huidos ambos de Lombardía hacia Trento con la expulsión de la familia Sforza del gobierno de la ciudad. Gerolamo Morone fue el hombre de confianza de Francesco II Sforza en el exilio trentino. Su hijo, Giovanni Morone, fue nombrado obispo de Modena en 1533, pasando posteriormente a las sedes de Novara y Ostia. Su actividad como diplomático se desarrolló en Alemania, donde ejerció su función como legado apostólico. En Roma mantiene contactos muy interesantes en el dinámico panorama cultural y teológico de mediados del XVI con el Cardenal Pole, Giulia Gonzaga e Vittoria Colonna, ésta última estrechamente vinculada con los jesuitas. Cfr. Fenlon D.,
} 

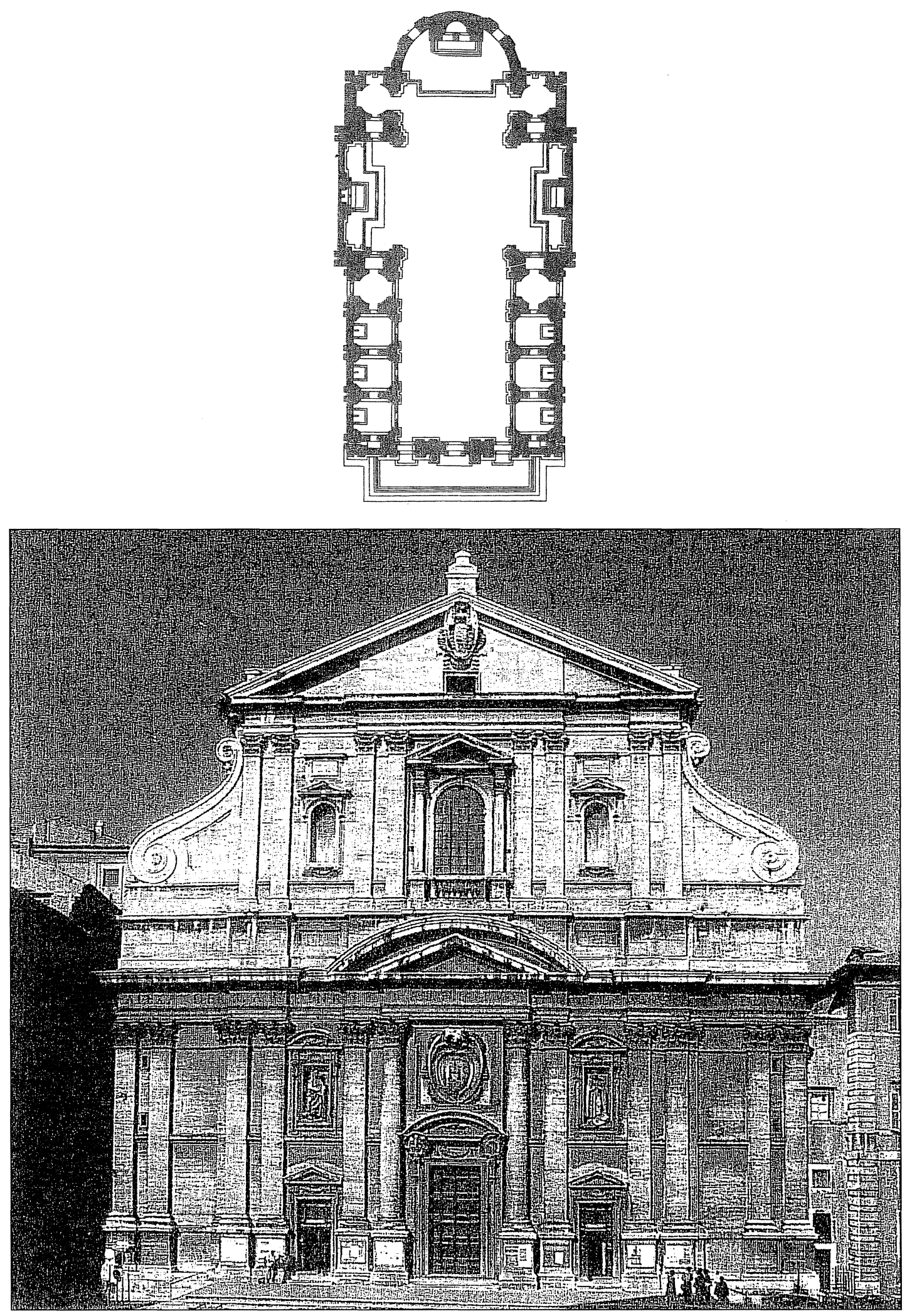

Figs. 1 y 2. Iglesia de Il Gesù de Roma. Planta y fachada.. 
En el documento aparecen citados únicamente dos jesuitas, el Padre Benedetto Giustiniani y el Padre Benedetto Palmio, que actuaron como celebrantes de sendas ceremonias religiosas en Il Gesù.

El Padre Palmio ${ }^{25}$ destacado miembro de la Compañía de Jesús, había iniciado su trayectoria en la Orden animado por el propio fundador y al Padre Laínez. Junto al cardenal Toledo, gozó de gran fama por su labor como orador, siendo invitado por el Cardenal Carlo Borromeo a Milán para predicar en la Catedral entre 1563 y 1566.

La estrecha relación del Padre Palmio con el cardenal Farnese se conoce a través de una carta ${ }^{26}$ enviada por el jesuita desde Venecia al cardenal en julio de 1583 en la que se citaba la finalización de las obras de $I l$ Gesù y la necesidad de un apoyo económico para construir la nueva Casa Profesa de la Compañía de Jesús en Roma.

Junto al Padre Palmio, en el documento se nombra un giovane di circa 30 anni conocido ya en el ambiente eclesiástico romano por sus aptitudes para la predicación: Benedetto Giustiniani ${ }^{27}$, jesuita sin vínculos familiares con la noble familia romana de los Giustiniani.

La trayectoria de este joven sacerdote, apenas había comenzado en 1576 cuando iniciaba sus predicaciones en las iglesias romanas mientras continuaba los pasos del Cardenal Toledo. Al final de su vida fue nombrado rector del Collegio Romano en 1599 y teólogo de la Sagrada Penitenciaria en 1606.

Los nombres de prelados señalados en líneas anteriores, son la prueba más evidente del poder de convocatoria que ejercía la Compañía de Jesús en sus inicios y la labor de promoción de ésta a través de una propaganda mediática ejercida desde los púlpitos con sermones dirigidos a estimular emociones e intelectos.

Por último, un acontecimiento casual señala nuevamente la importancia del inédito documento: la segunda celebración eucarística descrita en el documento, fechada el 25 de noviembre de 1576, onomástica de Santa Catalina de Alejandría, se anticipa en ocho años a la consagración oficial de $I l$ Gesù el 25 de noviembre de $1584{ }^{28}$.

Heresy and Obedience in the Tridentine Italy. Cardinal Pole and the Counter Reformation, University Press, Cambridge, 1972 y Firpo M. Il processo inquisitoriale del Cardinale Morone, Roma, 1981.

${ }^{25}$ Benedetto Palmio (Parma 1523-Ferrara 1598). De noble familia italiana, fue educado en el colegio de los jesuitas en Mesina, donde enseñó humanidades durante tres años. Ejerció el cargo de superintendente de la Orden en los colegios de Padua y Venecia entre 1557-1559 y fue provincial de Lombardía, además de asistente durante el generalato de Francisco de Borja. En 1573 se le acusó de haber participado en la conspiración ante Gregorio XIII para impedir la elección de un General español. Su último servicio a la Compañía fue su labor como mediador entre Clemente VIII y el P. General Claudio Aquaviva. Redactó un breve tratado sobre la predicación evangélica: De excellentia praedicationis evangelicae ed. Carlo Marcora en S.Carlo ed il gesuita Benedetto Palmio, publicado en Memorie Storiche della Diocesi di Milano, ${ }^{\circ} 16$ (1969) pp. 17-53.

${ }^{26}$ La irónica epístola se fecha el 16 de julio de 1583. La selección de dos fragmentos de la carta es suficiente para indicar el grado de amistad y complicidad entre ambos prelados: [… Con grandissima consolazione dell'anima mia ho inteso che la chiesa di V.S.Ill.ma già è finita et riesce un bellissimo tempio, con ammiratione e sodisfatione di tutti quei che

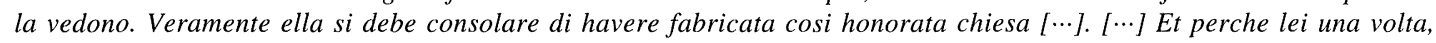
come credo si ricorderà, ridendo meco mi disse che cercassi un altro matto che ci facesse la casa, come ne havevamo trovato uno che ci fabricava la chiesa, venni a Venezia, dove si trovano huomini di omne natione, quae sub caelo est, et non ho potuto trovare matto simile a quello che ha fatto la chiesa nostra di Roma [ $\cdots$ ].

Cfr. Tacchi Venturi, Note storiche e topografiche di Roma nel sec. XVI. Le case abitate in Roma da S.Ignazio di Loyola, pp.335 y ss.

${ }^{27}$ Benedetto Giustiniani (Génova 1550-Roma 1622) Miembro de una distinguida familia genovesa, estudió en los colegios de jesuitas de Roma, Mesina y Toulouse. En 1578 mientras ejercía la docencia en el Collegio Romano escribió un memorial anónimo junto a otros prelados a Gregorio XIII contra la actuación del P. General Mercuriano. Predicador notable, preparó la oración fúnebre de Inocencio IX (1591) e influyó decisivamente en la anulación por parte del Papa del matrimonio entre Enrique IV de Navarra y Margarita de Valois. Escribió los comentarios a las epístolas del Nuevo Testamento.

${ }^{28}$ Cfr. Pecchiai, op.cit., pp. 350. En el apéndice documental de la monografía se publica la Autentica della Consacrazione della Chiesa del SS. Nome di Gesù in Roma escrita el 25 de noviembre de 1584 y conservada en el archivo de la iglesia. Este documento ya se ha citado a propósito de la presencia de Monseñor Odescalchi. 
Y como conclusión, la anotación manuscrita describe la perfecta conciliación de intereses con la estudiada proyección de la imagen de los jesuitas, jugando con la innovación arquitectónica y estética de su nuevo templo y la sistemática selección de conocidos nombres del panorama eclesiástico que indirectamente promocionaron el aparato ideológico de la Compañía de Jesús.

\title{
APÉNDICE DOCUMENTAL
}

\author{
BAV, Barb. Lat. 5377, ff 20r
}

\section{(al margen chiesa del Gesù)}

1576 alli 23 di novembre di sabbato a mezo giorno si mise da N.S.re Gregorio 13 in detta dal vespero fino al giorno seguente di notte, che fu la domenica avanti la prima dell'avvento, nella chiesa nova del Giesu, fatta da fondamenti dal Cardinale Farnese, come appare dalla tavola di pietra dentro, sopra le porte. La messa fu cantata in detta domenica da Mons. Odescalco vescovo e ci furono presenti molti cardinali, tra quali Farnese secondariamente, che per ordine era preceduto da Morone; l'uno, e l'altro vescovi delli VI [?]. La piazza era tanto piena de cocchi che non ci capevano; e pure è grandotta essa piazza, ch'è quella degli Altieri e sta davanti la prefata chiesa, la quale s'allargasi di sorte insieme con l'habitazione, che terrà loco grandissimo. Di già è si avanti, che sola impedisce la strada publica che non tocca la fabrica di S.Marco. Da qui innanzi dunque seguitarono quei religiosi di celebrarvi, havendo serrato la chiesa vecchia per dir così. Il seguente lunedì poi, alli 21, giorno di Santa Catarina, ci cominciò a predicar il padre Benedetto Giustiniano, giovane di circa 30 anni, valente certo, et il giorno dopo vespero il padre Benedetto Palmio, vecchio e valoroso. Questa chiesa è fatta la metà, o quasi, restando nel proprio loco, dove sarà la cuppola. Ci sono 6 capelle grandi, ciò̀ tre per banda; poi da una parte della strada è la porta piccola; dall'altra, ch'è di rincontro, è quella che va nell'habitatione de religiosi.

La facciata dinanzi è tutta di trivertino, con l'insegna del Giesu in mezo et in cima l'arme pur di trivertino del Cardinale prefato. Ci si legge anco nel (aparece tachado in) friscio di lettere negre Ascanius Farnesius S.R.E. Vicecancellarius fecit.1575. 